\section{Hiponatremia grave secundaria a la exposición a veneno de Phyllomedusa Bicolor (Rana Kambó). Caso clínico}

\author{
JUAN CAMPODÓNICO ${ }^{\mathrm{a}}$, PAULA AEDO ${ }^{\mathrm{a}}$, M. IGNACIA MONTANÉa \\ ALEJANDRA ROJAS ${ }^{\mathrm{a}}$, ANDREE AVEIGA ${ }^{1}$, LORENA SILVA ${ }^{1,2}$, \\ JUAN CARLOS RÍOS ${ }^{2}$, IVÁN SOLÍS ${ }^{3,4,5}$
}

\section{Severe hyponatremia secondary to Phyllomedusa bicolor (Kambó frog) poisoning. Report of one case}

Phyllomedusa bicolor or Kambo is a frog that lives in the Amazon rainforest. It can release through its skin a substance used in healing rituals that are common among South-American tribes, as well as in urban people of America and Europe. We report a 41-year-old female patient who, during a healing ritual consumed ayahuasca (a drink obtained from the mixture of Banisteriopsis caapi, Psychotria viridis and Mimosa hostilis) and 12 hours later received the poison of Kambo Frog (Phyllomedusa bicolor) on superficial right shoulder skin burns. The ritual included a minimum of six-liter water intake over a few hours period. She evolved with clouding of sensorium, motor agitation, frequent vomiting, and generalized tonic-clonic seizures. She presented lethargic to the emergency room, with a weak pupillary light reflex, generalized stiffness, moving all four limbs. Laboratory showed severe hyponatremia $(120 \mathrm{mEq} / \mathrm{L})$ and a creatine kinase level of 8,479 UI/L, that increased 107,216 IU/L within few days. An admission CT Brain scan was normal. The toxicological screening did not identify the presence of other substances. During hospitalization the patient developed severe psychomotor agitation controlled by a dexmedetomidine infusion, hyponatremia, low plasma osmolality (248 $\mathrm{mOsm} / \mathrm{kg}$ ), and disproportionately high urinary osmolality (448 $\mathrm{mOsm} / \mathrm{kg}$ ), suggestive of inappropriate antidiuretic hormone secretion syndrome (SIADH). With correction of hyponatremia, the patient gradually recovered consciousness. Rhabdomyolysis was assumed to be secondary to seizure and managed by volume and bicarbonate infusions with a positive response.

(Rev Med Chile 2019; 147: 935-939)

Key words: Amphibian Venoms; Banisteriopsis; Ceremonial Behavior; Hyponatremia; Phyllomedusa bicolor.

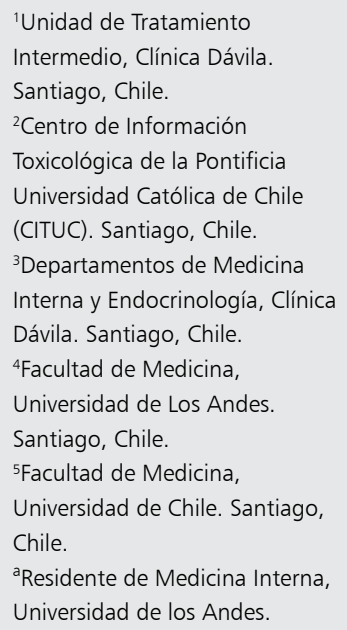

Recibido el 18 de diciembre de 2018, aceptado el 29 de agosto de 2019.

Correspondencia a

Dr. Iván Solís Opazo Departamento de Medicina Interna, Clínica Dávila. Recoleta 464, Santiago, Chile. isolis@davila.cl

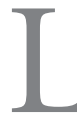

os rituales de sanación con medicinas alternativas son frecuentes. En Chile, se reporta que $71 \%$ de la población general alguna vez ha usado algún tipo de medicina alternativa ${ }^{1}$. Se ofrecen muchas de distinto tipo, sin dimensionar muchas veces los riesgos asociados a ellas.
El uso de veneno de la rana Phyllomedusa bicolor $(P B)$ o Kambó, es una práctica de medicina alternativa propia de culturas indígenas de la selva amazónica, que se ofrece en zonas urbanas alrededor del mundo. Se postula su utilidad para "purificar el cuerpo" y por medio de esto, tratar 
síntomas de varias enfermedades. La secreción excretada por las glándulas de la piel de $P B$ se extrae y es administrada durante un ritual vía subcutánea sobre quemaduras circulares. Previo se debe consumir importantes cantidades de agua ${ }^{2}$.

El siguiente caso clínico reporta el episodio de una paciente que sufre una intoxicación grave con el uso de estas sustancias con fines curativos, y busca alertar respecto de los riesgos asociados a este tipo de prácticas. $\mathrm{Al}$ respecto, se cuenta con el consentimiento informado por escrito de la paciente, cumpliendo con las normas de confidencialidad de nuestra institución.

\section{Caso clínico}

Paciente de 41 años femenina, con antecedentes de hipotiroidismo y un trastorno de personalidad limítrofe. Participa en un ritual chamánico de "purificación y sanación", durante el cual consume ayahuasca (bebida obtenida de la mezcla de partes de las plantas Banisteriopsis caapi, Psychotria viridis y Mimosa hostilis), posterior a lo cual consume al menos 6 litros de agua. Luego de algunas horas se le administra veneno de $P B$, sobre quemaduras cutáneas superficiales en hombro (Figura 1). Luego de dos horas de finalizado este ritual presenta vómitos frecuentes, diarrea profusa, compromiso de conciencia cuali-cuantitativo, y convulsiones tónico-clónicas. Dado el agravamiento de su compromiso de conciencia y una intensa agitación psicomotora, es llevada al Servicio de Urgencias.

$\mathrm{Al}$ ingreso, transcurridas aproximadamente $12 \mathrm{~h}$ del ritual, la paciente se encontraba agitada, signos vitales estable (FC: $80 \mathrm{lpm}$, PA: $150 / 80 \mathrm{mmHg}$ ), describiéndose su estado de conciencia como sopor superficial, al examen pupilar con midriasis y reflejo fotomotor débil, movilizando las 4 extremidades. $\mathrm{Al}$ ingreso, destaca hiponatremia severa $120 \mathrm{mEq} / \mathrm{L}$, hipocalemia $3,5 \mathrm{mEq} / \mathrm{L}$, alcalosis respiratoria aguda (PH: 7,52, HCO3: 19,9, PCO2: 24,9), niveles de creatinquinasa y lactato venoso elevados ( $8.479 \mathrm{U} / \mathrm{L}$ y $25 \mathrm{mg} /$ $\mathrm{dL}$, respectivamente), aumento de LDH 9.313 U/L, elevación de transaminasas AST de 1.387 U/L, ALT $318 \mathrm{U} / \mathrm{L}$, con un screening toxicológico en orina positivo sólo para benzodiacepinas (fueron usadas para manejo de la agitación). Una tomografía computada de cerebro sin contraste no mostraba lesiones agudas. El cuadro se interpreta como encefalopatía tóxico metabólica secundaria a uso de veneno de rana Kambó, por lo cual se decide su hospitalización en la unidad de paciente crítico.

La hiponatremia severa del ingreso se asoció a osmolalidad plasmática baja $(248 \mathrm{mOsm} / \mathrm{kg})$, y osmolalidad urinaria desproporcionadamente elevada (448 mOsm $/ \mathrm{kg})$, sugerente de secreción inapropiada de $\mathrm{ADH}$ (SIADH). Se descartaron razonablemente otras causas de hiponatremia, como hipotiroidismo y falla adrenal aguda, al presentar hormona tiroestimulante y cortisol plasmático matinal dentro de rangos normales $(1,28 \mathrm{uUI} / \mathrm{ml} \mathrm{y}$ 9,05 ug/dl respectivamente), además, se descartó la deshidratación secundaria a vómitos como causa de hiponatremia debido a que esta persiste pese a volemización inicial (más de 2 litros de solución salina), y no hay elevación de nitrógeno ureico ni uricemia en los exámenes iniciales. Se manejó con restricción de ingesta de agua y solución fisiológica. La velocidad de corrección del sodio fue adecuada (aproximadamente $0,22 \mathrm{meq} / \mathrm{lt} / \mathrm{h}$ en las primeras $72 \mathrm{~h}$ ).

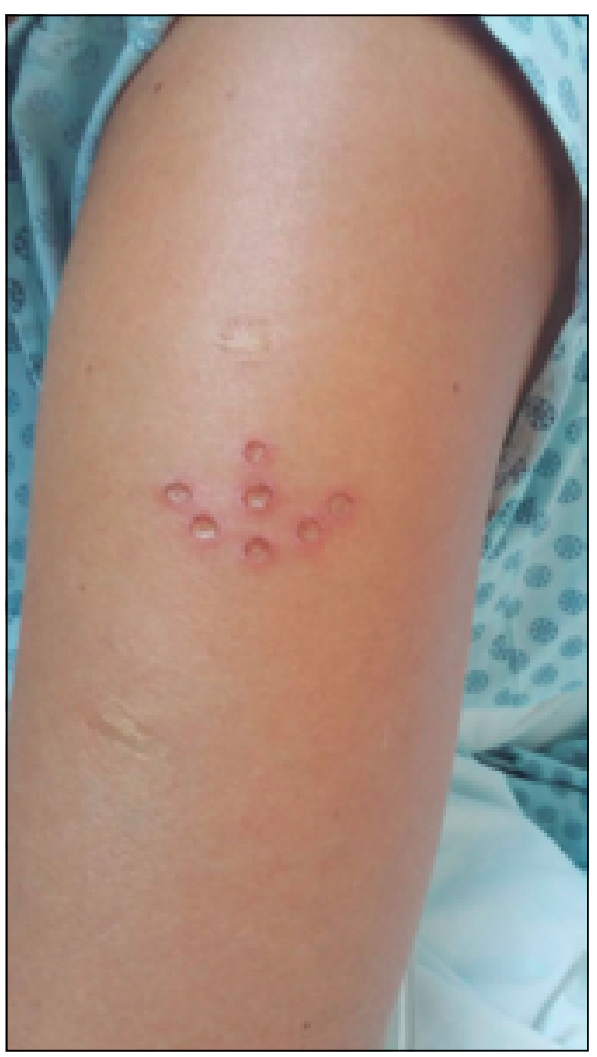

Figura 1. Lesiones típicos de rituales de Kambó. 
Tabla 1. Evolución de exámenes de laboratorio

\begin{tabular}{|c|c|c|c|c|c|}
\hline Fecha & Ingreso & Día 1 & Día 3 & Día 5 & Día 7 \\
\hline Hto (\%) /Hb (g/dL) & & $37 / 13$ & & $32,3 / 11,4$ & $35,5 / 12,3$ \\
\hline $\mathrm{RGB}\left(\mathrm{x} 10^{3} / \mathrm{mm}^{3}\right) / \mathrm{PCR}(\mathrm{mg} / \mathrm{dL})$ & & $11,6 / 5,5$ & & $6,0 / 8,2$ & $5,7 /-$ \\
\hline BUN/Crea (mg/dL) & & $5,6 / 0,6$ & $-/ 0,5$ & $-/ 0,5$ & \\
\hline $\mathrm{Na} / \mathrm{Cl}(\mathrm{meq} / \mathrm{L})$ & $120 /-$ & $125 / 94$ & $136 / 106$ & $137 / 106$ & $137 / 03$ \\
\hline $\mathrm{LDH}(\mathrm{U} / \mathrm{L})$ & & 9313 & 4086 & 1400 & 784 \\
\hline GOT/GPT (U/L) & & $1.387 / 318$ & $1.351 / 386$ & $1.202 / 508$ & $720 / 477$ \\
\hline $\mathrm{GGT} / \mathrm{FA}(\mathrm{U} / \mathrm{L})$ & & $21 / 67$ & $-/ 49$ & $-/ 47$ & $22 / 51$ \\
\hline BiliT/Bilil (mg/dL) & & $1,3 / 1,3$ & $0,5 /-$ & $0,3 /-$ & $0,3 / 0,2$ \\
\hline $\mathrm{TP}(\%)$ & & 77 & & & \\
\hline CKT (U/L) & 8.479 & 93.359 & 91.738 & 43.188 & 4.968 \\
\hline Ac. Úrico (mg/dL) & & 2,6 & & & \\
\hline Toxicológico orina & & $(+) B Z D$ & & & \\
\hline Osmolalidad plasma (mOsm/kg) & & 259 & 294 & & \\
\hline Osmolalidad Urinaria (mOsm/kg) & & 448 & 448 & & \\
\hline Lactato Venoso (mg/dL) & 25 & & & & \\
\hline
\end{tabular}

*Abreviaciones: Hto: Hematocrito; Hb: Hemoglobina; RGB: Recuento Glóbulos Blancos; BUN: Nitrógeno Ureico en Sangre; Crea: Creatinina en Sangre Na: Sodio sérico; Cl: Cloro Sérico; LDH: Lactacto deshidrogenada; GOT: alanina aminotransferasa; GPT: glutamato-piruvato transaminasa; GGT: gama glutamil transpeptidasa; FA: Fosfatasa Alcalina; BiliT: bilirrubina total; Bilil: Bilirrubina indirecta; TP: tiempo de protombina; CKT: creatina quinasa total; BZD: benzodiazepinas.

La agitación psicomotora requirió medidas como contención mecánica y farmacológica con infusión de dexmedetomidina hasta $0,4 \mathrm{ug} / \mathrm{kg} /$ min. La ausencia de signos de focalidad neurológica, asociado a una resonancia magnética de encéfalo normal y un electroencefalograma con lentitud de ondas fueron compatibles con encefalopatía tóxico-metabólica.

La significativa elevación de niveles de creatinquinasa, hasta valores de 107.218 U/L, se interpretó como rabdomiólisis mixta, probablemente secundaria a toxicidad directa del tóxico, como también secundario al cuadro convulsivo. Fue manejada con aporte de volumen y bicarbonato de sodio de acuerdo a $\mathrm{pH}$ urinario con respuesta favorable, sin deterioro de la función renal. Las transaminasas persisten elevadas, con lento descenso, fueron interpretadas en contexto de rabdomiólisis. No presentó elementos de falla hepática, pese a la alteración de la AST/ALT probablemente en contexto de hepatitis secundaria.

La evaluación psiquiátrica descartó que la in- toxicación estuviese en contexto de un intento de autolisis. Luego de una semana de hospitalización, la paciente se encontraba con resolución de sus conflictos de ingreso por lo que se decide su alta y control ambulatorio.

\section{Discusión}

En el caso expuesto, se presenta el cuadro clínico de una paciente con compromiso de conciencia grave, asociado a convulsiones en relación a la exposición aguda al veneno de $P B$ y ayahuasca, ingeridas en el contexto de un ritual chamánico.

Las sustancias que contiene el veneno de $P B$ son más de cien péptidos activos, la mayoría derivado de un grupo de precursores conocidos como preprodermorfinas/dermaseptinas. Los péptidos dermorfinas y deltorfinas son agonistas opioides $\mathrm{Mu}$, que actúan sobre los receptores opioides $\mathrm{Mu}$ y Delta presentes en sistema nervioso central implicados en los efectos analgesicos, disforicos y de 
dependencia propios del péptido. Las deltorfinas tienen alta afinidad con los sitios de unión de receptores opioides delta ${ }^{3,4}$. Sauvagine es un neuropéptido con efecto como el de hormona liberadora de corticotropina, que en ratas ha demostrado que produce un aumento en la liberación de cortisol, catecolaminas y glucosa, mayor liberación de beta endorfinas, parece tener efecto hipotensor y causar potente taquicardia compensatoria y diarrea, ésta última posiblemente secundario a vasodilatación intestinal ${ }^{5}$. A nivel periférico la Phyllocaerulina parece causar efectos gastrointestinales tales como náuseas y vómitos, la Phyllomedusina es una taquinina que causa vasodilatación, contracción del músculo liso y la Phyllokinina es una bradiquinina que relaja el músculo liso arterial ${ }^{6}$. No encontramos estudios que muestren el mecanismo por el cual este tóxico podría desencadenar un SIADH.

En el caso de la paciente, la hiponatremia podría explicarse por el efecto del veneno de $P B$ a nivel central, el cual precipitaría un SIADH, agravado por una polidipsia intensa propia del ritual, como también de los vómitos que presento inicialmente. Estos últimos dos factores se corrigieron inicialmente, y la hiponatremia persistió. Su rápida instalación, puede explicar el compromiso de conciencia y las convulsiones que se presentaron en este caso. La rabdomiólisis probablemente mixta, produce elevación brusca de enzimas presentes en células musculares, como creatinquinasa, las aminotransferasas y lactato deshidrogenasa en plasma. Una de sus consecuencias más serias es la falla renal aguda, que afortunadamente no se presentó durante la evolución. El consumo de ayahuasca de la paciente no parece haber tenido significancia en la evolución de este caso, ya que está descrito el tiempo de evolución de síntomas por consumo de ayahuasca los cuales no deberían estar a los $360 \mathrm{~min}$ posteriores a la ingesta ${ }^{7}$.

La forma de presentación más habitual descrita de intoxicación por Kambó es un cuadro de náuseas, dolor abdominal y vómitos frecuentes, habitualmente autolimitados ${ }^{8}$. También se reportan complicaciones graves de esta práctica. Uno de los casos reportados presentó compromiso de conciencia y convulsiones asociadas a un SIADH ${ }^{9}$. En otro reporte, un paciente desarrolla una hepatitis aguda tóxica secundaria a la utilización de esta sustancia ${ }^{10}$. Existe un reporte de fallecimiento por uso de veneno de $P B^{11}$.

En nuestro país, según la base de datos del
Centro de Información Toxicológica de la Universidad Católica (CITUC), se han reportado un total de 7 casos de exposición aguda a Kambó entre 2014 y primer semestre de 2018. Todos ellos en el contexto de exposición por "rituales de sanación". Todos adultos de entre 21 a 63 años, con una distribución uniforme según sexo. Los síntomas más frecuentes reportados son vómitos y compromiso de conciencia. Otros efectos clínicos vinculados son: agitación psicomotora, psicosis, alucinaciones, fiebre, cefalea, náuseas. Los llamados realizados al Centro provinieron desde centros asistenciales y en un solo caso el llamado es realizado desde el domicilio, por parte de un familiar del individuo expuesto. Cabe destacar que los pacientes consultan en servicios de urgencia desde las $12 \mathrm{~h}$ post exposición ${ }^{12}$.

Las limitaciones de este reporte son en primer lugar que el paciente no fue recibido en una primera instancia en nuestro centro, por lo que toda la información de la presentación inicial fue obtenida de informes de traslado. También no fue posible medir metabolitos del veneno de $P B$ en sangre como marcadores de toxicidad, ya que esta técnica no está disponible.

Es importante destacar que en Chile no es difícil acceder a este tipo de prácticas. A través de internet son múltiples los centros que ofrecen sesiones de Kambó. En ellas se plantea como una alternativa de medicina natural, que ofrece respuesta a múltiples problemas de salud y a un precio accesible ${ }^{13}$. Esto induce a muchos pacientes a recurrir a ella sin saber los potenciales riesgos a los cuales se someten.

Este caso pone de manifiesto los riesgos asociados al consumo y uso inadecuado de estas sustancias como práctica de medicina alternativa. Creemos importante generar conciencia acerca de esta situación y a hacer un llamado a la rigurosa fiscalización de estas prácticas en nuestro medio.

\section{Referencias}

1. Organización Mundial de la Salud. Estrategia de la OMS sobre medicina tradicional 2002-2005. Ginebra, Suiza: World Health Organization 2002. http://apps.who.int/ medicinedocs/es/d/Js2299s/.

2. den Brave P, Bruins E, Bronkhorst M. Phyllomedusa bicolor skin secretion and the Kambô ritual. J Venom Anim Toxins Incl Trop Dis 2014; 20 (1): 40.

3. Dupin S, Tafani JA Mazarguila H, Zajaca JM. [125I] 
[D-Ala2]deltorphin-I: A high affinity, delta-selective opioid receptor ligand. Peptides 1991; 12 (4): 825-30.

4. Mizoguchia H, Bagetta G, Sakurada T, Sakurada S. Dermorphin tetrapeptide analogs as potent and long-lasting analgesics with pharmacological profiles distinct from morphine. Peptides 2011;32(2): 421-7.

5. Brown MR, Fisher LA, Spiess J, Rivier J, Rivier C, Vale W. Comparison of the biologic actions of corticotropin-releasing factor and sauvagine. Regul Pept 1982; 4 (2): 107-14.

6. Erspamer V, Erspamer GF, Severini C, Potenza RL, Barra D, Mignogna G, et al. Pharmacological studies of 'sapo'from the frog Phyllomedusa bicolor skin: a drug used by the Peruvian Matses Indians in shamanic hunting practices. Toxicon 1993; 31(9): 1099-111.

7. Riba J, Valle M, Urbano G, Yritia M, Morte A, Barbanoj MJ. Human pharmacology of ayahuasca: subjective and cardiovascular effects, monoamine metabolite excretion, and pharmacokinetics. J Pharmacol Exp Ther 2003; 306(1): 73-83.

8. Kumachev A, Zipursky JS, Weinerman AS, Thompson M. Poisoning from the Kambô ritual. CJEM 2018; 20
(6): 962-4.

9. Leban V, Kozelj G, Brvar, M. The syndrome of inappropriate antidiuretic hormone secretion after giant leaf frog (Phyllomedusa bicolor) venom exposure. Toxicon 2016; 120: 107-9.

10. Pogorzelska J, Łapiński TW. Toxic hepatitis caused by the excretions of the Phyllomedusa bicolor frog-a case report. Clin Exp Hepatol 2017; 3 (1): 33.

11. Aquila I, Gratteri S, Sacco M, Fineschi V, Magi S, Castaldo P, et al. The Biological Effects of Kambo: Is There a Relationship Between its Administration and Sudden Death? J Forensic Sci 2018; 63(3): 965-8.

12. Casos de exposición a Rana Kambó (Phyllomedusa bicolor) reportados al centro de Información Toxicológica de la Facultad de Medicina de la Pontificia Universidad Católica de Chile (CITUC) entre el año 2014 al primer semestre del año 2018. (2018). [ebook] Available at: http://cituc.uc.cl/images/articulos/casos-cituc-rana-kambo.pdf [citado el 9 de julio de 2019].

13. https://www.medicinakambo.cl/beneficios/ [Internet]. Santiago: Kambô Santiago; c2017 [citado el 27 de junio de 2018]. Disponible en: https://www.medicinakambo.cl/. 